\title{
Hypertension management in 2030: a kaleidoscopic view
}

\author{
Spoorthy Kulkarni $\mathbb{D}^{1}$
}

Received: 21 July 2020 / Revised: 4 August 2020 / Accepted: 20 October 2020 / Published online: 2 November 2020

(c) The Author(s), under exclusive licence to Springer Nature Limited 2020

\begin{abstract}
The last decade has witnessed the healthcare system going paperless with increased use of electronic healthcare records. Artificial intelligence tools including smartphones and smart watches have changed the landscape of day-to-day lives. Digitisation, decentralisation of healthcare and empowerment of allied healthcare providers and patients themselves have made shared clinical decision-making a reality. The year 2020 quickly turned into an unprecedented time in our lives with the entry of COVID-19. Amidst a pandemic, healthcare systems rapidly adapted and transformed, and changes that otherwise would have taken a decade, took a mere few weeks (Webster, Lancet 395:1180-1, 2020). This essay reviews evidence of transformation in the realm of hypertension management, namely diagnosis, lifestyle changes, therapeutics and prevention of hypertension at both individual and population levels, and presents an extrapolation of how this transformation might shape the next decade.
\end{abstract}

\section{Introduction}

The last decade has witnessed the healthcare system going paperless with increased use of electronic healthcare records. Artificial intelligence tools including smartphones and smart watches have changed the landscape of day-today lives. Digitisation, decentralisation of healthcare and empowerment of allied healthcare providers and patients themselves have made shared clinical decision-making a reality. The year 2020 quickly turned into an unprecedented time in our lives with the entry of COVID-19. Amidst a pandemic, healthcare systems rapidly adapted and transformed, and changes that otherwise would have taken a decade, took a mere few weeks [1]. This essay won the first place in the British and Irish Hypertension Society Stanley Peart Essay competition (Fig. 1). The essay itself reviews evidence of transformation in the realm of hypertension management, namely diagnosis, lifestyle changes, therapeutics and prevention of hypertension at both individual and population levels, and presents an extrapolation of how this transformation might shape the next decade. It is our onus to ensure that adaptations made

Spoorthy Kulkarni

Spoorthyburli@gmail.com

1 Cambridge University Hospitals NHS Foundation Trust, Cambridge, UK to healthcare delivery as a result of the pandemic remain beneficial to patients and continue to promote best practice.

\section{Burden of hypertension}

Raised blood pressure (BP) is the leading global risk factor for cardiovascular mortality and morbidity. However, even in 2020, it is not uncommon to encounter patients who have never had a single BP measurement until the presentation of a disabling stroke. Unknown, untreated and inadequately treated hypertension make it a silent killer disease. As per American Heart Association policy predictions, $\approx 41.4 \%$ of US adults will develop hypertension by 2030 [2]. Currently, less than one in five individuals with hypertension are at target. One of the WHO global targets is to reduce the prevalence of hypertension by $25 \%$ by 2025 from the year 2010 [3]. A paper in Lancet [4] claimed that this would be unachievable in low-income countries, for instance in south Asia and sub-Saharan Africa, due to opposite trends. This calls for urgent action.

\section{Current versus novel pharmacotherapies}

In principle, the management of hypertension has not changed in the last two decades, broadly involving pharmacotherapy and lifestyle modification. The first anti- 
Fig. 1 BIHS Stanley Peart essay award. Dr. Spoorthy

Kulkarni: Winner of BIHS

Stanley Peart Essay 2020.

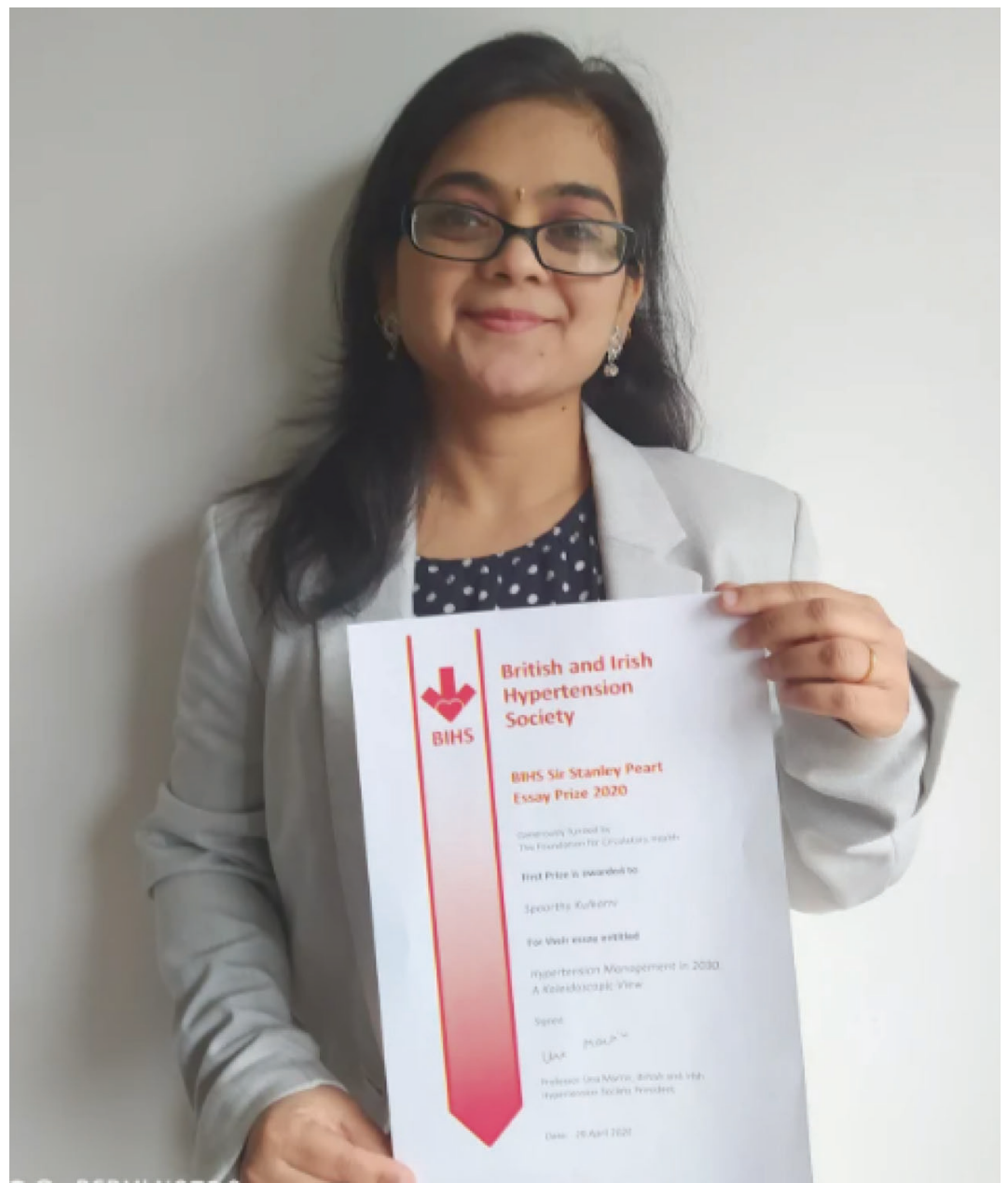

hypertensive medication to be trialled was pentaquine, in 1946. Diuretics and beta-blockers revolutionised hypertension management in the 1960s, and the 1990s saw the emergence of calcium channel blockers, angiotensin converting enzyme inhibitors (ACEi) and angiotensin receptor blockers (ARB), the current first-line therapies. These antihypertensive medications reduce the risk of myocardial infarction by $20-25 \%$, stroke by $30-40 \%$ and heart failure by $50 \%$ [5]. Medications based on various converging pathways such as direct renin inhibitors, nitric oxide inhibitors, endothelin antagonists and imidazoline inhibitors were trialled, however, none surpassed the current first-line therapies in efficacy and safety. The strength of current antihypertensive medications is their reliability and affordability. However, they do not reverse the pathophysiological complications of hypertension and hence therapy remains lifelong. It is also difficult to predict which drug will be most effective on an individual level. This has driven an exhaustive search for personalised and novel treatment strategies.
Hypertension has emerged as a major risk factor of mortality with COVID-19 disease. Use of angiotensin system inhibitors (ASI) for hypertension in COVID-19 patients created a theoretical controversy early on in the pandemic. The angiotensin converting enzyme 2 (ACE2) enzyme acts as a receptor for the coronavirus in the epithelial cells on the lung and ACE2 levels are increased by ASIs. Hence, it was hypothesised that ASIs may predispose to increased susceptibility to COVID-19 infection and thereby increase morbidity [6]. Large observational cohort studies in COVID-19 have now shown that ACEi/ARBs do not raise the risk of mortality in hypertensive patients and there is a possibility of a beneficial role. National and international guidelines suggest to continue ASIs for hypertension at this point in time.

Under the umbrella of resistant hypertension, management of hyperaldosteronism has seen advances in diagnosis, with better understanding of adrenal venous sampling and emergence of newer imaging techniques such as metomidate-labelled positron emission tomography [7]. 
Surgery is the treatment of choice in unilateral hyperaldosteronism. Device therapies such as renal denervation and baroreceptor modulation have not proven to be effective in mitigation of hypertension in randomised control trials (RCTs), however, future RCTs might benefit from stratification of patients, accessibility, and improved participation in trials. There are currently 7944 trials focussed on hypertension registered on ClinicalTrials.gov, as of 22 May 2020. This is possibly going to grow further with increased application of newer technologies including genomics, nutrigenomics, nanotechnology and chronotherapeutics.

\section{Genomics of hypertension}

Identification of genetic targets of the renin-angiotensin system in rat models was published almost 19 years ago [8]. Forty per cent of FDA-approved drugs in 2018 have the potential for personalised therapy based on genomics. The explosion of data and plummeting costs of gene sequencing has led to an enormous knowledge base of monogenic and polygenic factors that determine BP regulation and variability in drug responses. Over 500 single nucleotide polymorphisms associated with BP are now recognised. Genomics has the potential for stratified and personalised therapies. The AIM HY-INFORM trial is one such trial designed to personalise treatment based on self-defined ethnicity [9], using first-line therapies.

\section{Gene therapy-small interfering RNA (siRNA)}

Gene therapy refers to gene silencing of receptors, which regulate an outcome. Advances in biomedical and biophysics sectors have enabled major surges in the development of gene therapies. Indeed, 2018 saw the first approval of siRNA, patisiran (ONPATTRO), an siRNA encapsulated in lipid nano particle, by the FDA [10]. Inclisiran (siRNA-targeted therapy) is currently under investigation for hypercholesterolaemia in a large phase 3 trial after success in a phase 2 trial [11]. A recent study showed efficacy of siRNA targeting angiotensinogen in spontaneously hypertensive rats, comparable to ACEi and ARB [12]. Both AT1147siRNA [13] and siTRPC3 [14] silence AT1a, interfering RNA targeting subtype of angiotensin receptor and calcium permeable transient receptor potential channel, respectively, have the potential to develop into treatment options for hypertension. Indeed, some of the initial issues in target selectivity and stable expression of gene therapy seem to find resolutions.

\section{Chrono-therapeutics and e-trials}

It has been established that night-time hypertension increases cardiovascular risk. The recently published Hygia study showed that routine night-time dosing of medications improved night BP and reduced cardiovascular death (hazard ratio: $0.44(0.34-0.56)$ ) [15]. TIME is another trial studying chrono-therapeutics. The novelty of this study includes an electronic case report form and record linkage to identify potential endpoints [16]. Planned and future trials are likely to incorporate this design in the current pandemic and post-pandemic era, for the benefits of cost reduction and participant-friendly aspects.

Another major leap in the pharmaceutical industry is the use of three-dimension (3D) printing technology. Spritam (levetiracetam) was the first FDA-approved medication that was built via 3D printing in 2015. The advantages of this technology are the possibility of tailored treatment, incorporating complex drug release profiles [17]. The fusion of nanomedicines, chrono-therapeutics and 3D printing may revolutionise treatment in a few decades. This could enable drug release at the point of rise in $\mathrm{BP}$ in a predictable manner, leading to precise, point of care, individualised BP management.

\section{Hypertension prevention}

It is indisputable that lifestyle changes play an important role in hypertension prevention and management. A metaanalysis showed that mean weight reduction of $-5.1 \mathrm{~kg}$ was achieved by means of energy restriction, and with or without increased physical activity, reduction in systolic BP by $-4.4 \mathrm{~mm} \mathrm{Hg}$ and diastolic BP by $-3.6 \mathrm{~mm} \mathrm{Hg}$ was noted [18]. Plant-based diets have shown to also reduce BP significantly, in various cohort studies $[19,20]$. The benefits of these diets maybe postulated to be due to effective modulation of gut microbiota, thereby reducing adverse metabolic patterns [21]. This aim is shared by other research modalities such as faecal transplant [22]. Yoga and mindfulness training have also proven to have modest effects on BP in small studies [23, 24]. A package of multi-component intervention based on promoting increased exercise levels, DASH diet, reducing salt intake and weight loss reduced hypertension prevalence by an odds ratio of $0.77(95 \% \mathrm{CI}$ : 0.62-0.97) in comparison to advice alone [25]. Health interventions using mobile technology, that is mobile health, are increasingly used to provide patients and healthcare providers tools to deliver lifestyle interventions. In a small study, mobile-based self-management support system significantly reduced systolic $(-7 \mathrm{~mm} \mathrm{Hg})$ and diastolic (-4.9) BP over 8 weeks [26]. In addition, it also improved adherence to medications. Digital applications 
targeting stress, anxiety, exercise, counselling, yoga and mindfulness are widely accessible. A recent phase 2 trial (REACH study) showed the effectiveness of "e" counselling in reducing cardiovascular risk [27].

\section{Adherence}

Recognising psychological complexities of behaviour modification and medication taking, and their impact on outcomes of hypertension management, both clinically and in trials, has led adherence testing evolve from the humble pill count to use of sophisticated biomarkers such as urine- or plasma-based biochemical adherence screens [28]. Medications with inbuilt ingestible sensor to detect adherence may become a commonality in the near future [29] (Proteus Digital Health, Inc., Redwood City, CA, USA).

\section{Diagnosis of hypertension}

Appropriate management of hypertension is dependent on accurate BP measurement. The last decade ushered in ambulatory and home BP monitors and these measurements are widely used for diagnosis and assessing treatment responses. The mercury sphygmomanometer is now almost obsolete; however, it is highly unlikely that the sphygmomanometer cuff is going to be. Nevertheless, there are promising developments of newer non-invasive and "cuffless" monitoring devices [30]. COVID-19 fast-tracked growth in areas of healthcare delivery, mainly telemedicine. Changes, such as use of video chats to communicate with patients, and reliance on home BP monitoring, are already integrated into standard of care. By 2030, reliable, accurate, validated and user-friendly wearable devices such as smart watches and skin patches, seamlessly providing ambulatory BP data to healthcare providers, might turn into reality.

\section{Hypertension management: population- level strategies}

Crucial healthcare policy changes in education, awareness and diagnosis of the burden of hypertension are likely to bring about the most impact on reduction of that burden, on a larger scale. Patient empowerment through education delivered via nursing care and pharmacies will help to decentralise focus from tertiary healthcare settings to the community. Simple strategies, such as opportunistic screening of BP, education personalised to the patient and linking up information are urgently needed. Community pharmacists are well-placed to deliver these strategies, and with adequate, ongoing support of appraisal and education, pharmacists could become first-line providers in the management of hypertension [31].

Change4life, a programme established in the UK launched more than 10 years ago, promotes healthy lifestyle in children. Since then, a national diet and nutrition survey showed that this campaign led to significant reductions in consumption of sugars from ages four to ten [32]. Education at school level is crucial, making children the frontiers of change in lifestyle, as evident from interventions directed towards climate change.

May Measurement Month [33], an effective global campaign conducted annually, is another successful campaign to promote awareness of the importance of BP. In 2018 alone, providers detected 224,285 individuals requiring treatment, and 111,214 inadequately treated individuals. Extrapolating from this campaign, the introduction of BP kiosks or stations where BP can be checked on demand, using automatic monitors, and without the need for long queues or time spent awaiting a consultation, could be considered. However, widespread implementation of such a strategy at the level of primary care is not yet feasible [34].

In low-income countries, the focus should primarily include improving access to medicines and technologies and a system for monitoring, along with a systematic approach to management. In line with this, the WHO led Global Hearts Initiative included a technical package called HEARTS, which includes essential modules for a strategic approach [35]. This has already been implemented in 15 countries and initial successes are evident (Fig. 1).

\section{Summary}

Adequate hypertension management will undoubtedly have one of the biggest impacts on saving lives in the coming years. Table 1 and Fig. 2 summarise the key themes visited in this essay. A systems-based approach, with a focus on prevention of hypertension by increased awareness and education, early detection and active participation of patients in management, appropriate use of digitisation and integration of pharmacogenomics, will make personalisation of therapy a reality. Quite possibly, hypertension clinic might be unrecognisable in 2030. However, as evidenced amidst a pandemic, the patient-physician relationship will remain the most crucial factor in the healthcare system. The future is usually a combination of yesterday, a vision and concerted efforts to make that vision a reality. It is probably prudent, therefore, to live by Albert Einstein's thoughts on future, "I never think of future, it comes soon enough". 
Table 1 Summarises the possibilities of various changes in hypertension management.

\begin{tabular}{ll}
\hline Questions & Contemplation
\end{tabular}

Will BP monitors change in size and shape?

What will be the recommendation for patients with pre-hypertension or other cardiovascular risk factors such as diabetes?

Who will be the first point of call for patients, if patient's smart apps/home BP machines reveal high BP values?

Will it be common for clinicians to see hypertensive patients physically in clinic?

Will there be community-based BP stations similar to swabtesting drop ins?

Will medications change?

Will management protocols/guidelines change?

What aspects of medication-related advice are likely to change?

What aspects of medication production are likely to change?

Will clinical trial designs change?

Who will be the primary focus of education on lifestyle modifications?

How will education on lifestyle be provided for patients?
Validated wrist monitors will be routinely used, and they will get smaller. Sensor-based technology and wearables may become standard.

A package of lifestyle modifications including exercise, plant-based therapy, salt reduction, yoga and mindfulness training. Faecal transplant/gut immune modulation is an emerging theme.

Community pharmacists.

Very likely, however, a subset of patients may be reviewed virtually at regular intervals.

Very likely. BP readings from kiosks may also be integrated into electronic healthcare records.

Unlikely, although targeted genetic therapies may be tested.

Likely to be more patient centred and personalised.

To take medications at night-time, where feasible.

Drug delivery systems with use of nanotechnology and possibly 3D printing.

Medications may be inbuilt with sensors to detect adherence.

More pragmatic, adaptive and e-trials may be embraced to answer some research questions.

Paediatric age group.

Standardised electronic counselling via digital/AI applications.
Fig. 2 Highlights the key areas which can transform hypertension management.

Maximum impact can be brought about by overlap of efforts working towards common goals of systems strategy towards precision medicine. Adapted from Dzau and Balatbat [36]

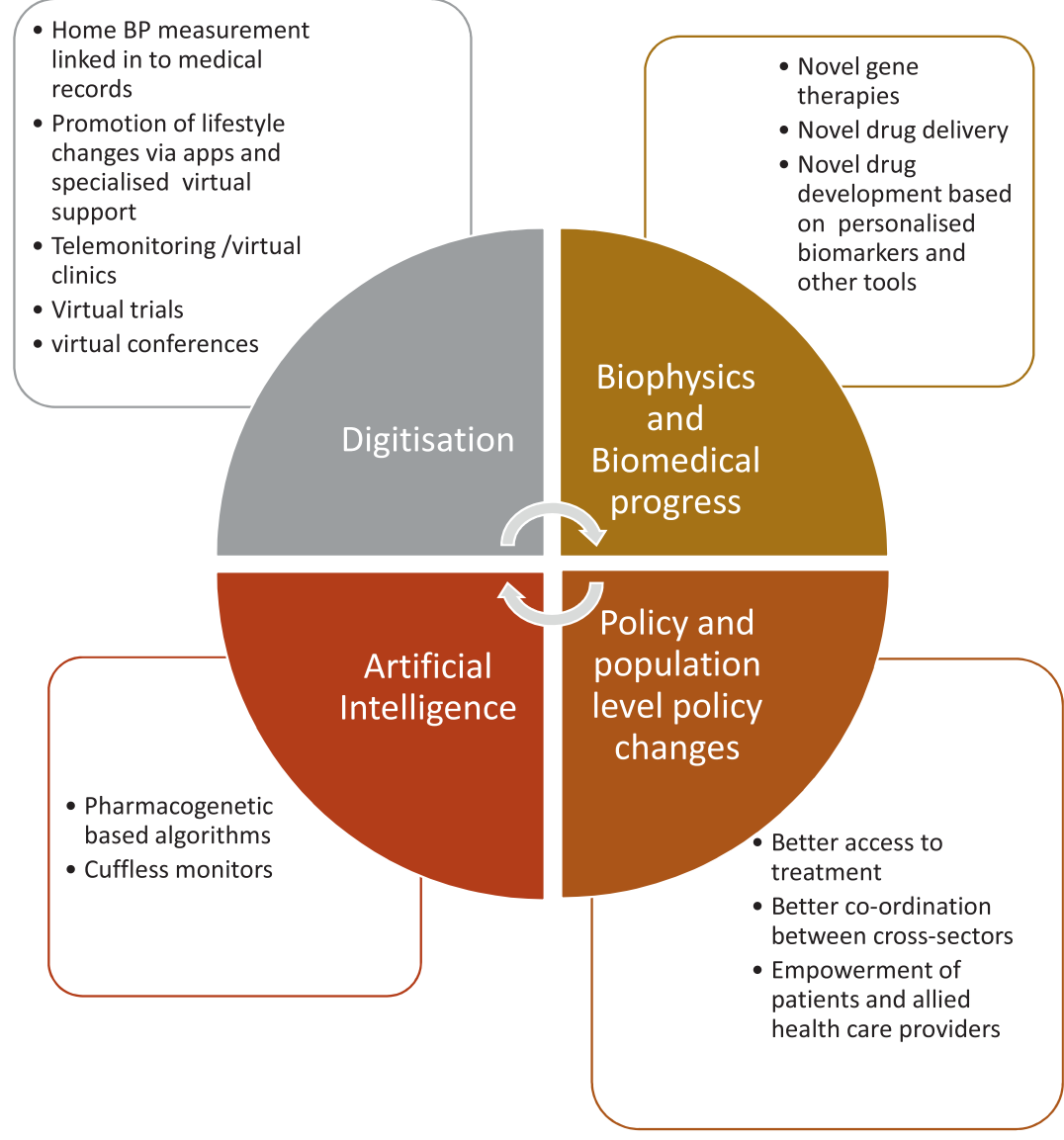




\section{Compliance with ethical standards}

Conflict of interest The author declare no conflict of interest.

Publisher's note Springer Nature remains neutral with regard to jurisdictional claims in published maps and institutional affiliations.

\section{References}

1. Webster P. Virtual health care in the era of COVID-19. Lancet. 2020;395:1180-1.

2. Heidenreich Paul A, Trogdon Justin G, Khavjou Olga A, Butler J, Dracup K, Ezekowitz Michael D, et al. Forecasting the future of cardiovascular disease in the United States. Circulation. 2011;123:933-44.

3. WHO. https://www.who.int/news-room/fact-sheets/detail/hypertension (2019).

4. Zhou B, Bentham J, Di Cesare M, Bixby H, Danaei G, Cowan MJ, et al. Worldwide trends in blood pressure from 1975 to 2015: a pooled analysis of 1479 population-based measurement studies with 19.1 million participants. Lancet. 2017;389:37-55.

5. Turnbull F, Neal B, Ninomiya T, Algert C, Arima H, Barzi F, et al. Blood Pressure Lowering Treatment Trialists' Collaboration. Effects of different regimens to lower blood pressure on major cardiovascular events in older and younger adults: meta-analysis of randomised trials. BMJ. 2008;336:1121-23.

6. Kai H, Kai M. Interactions of coronaviruses with ACE2, angiotensin II, and RAS inhibitors-lessons from available evidence and insights into COVID-19. Hypertens Res. 2020;43:648-54.

7. Wolley MJ, Stowasser M. New advances in the diagnostic workup of primary aldosteronism. J Endocr Soc 2017;1:149-61.

8. Pachori AS, Huentelman MJ, Francis CS, Gelband CH, Katovich MJ, Raizada MK. et al. The future of hypertension therapy: sense, antisense, or nonsense?. Hypertension. 2001;37:357-64.

9. Mukhtar O, Cheriyan J, Cockcroft JR, Collier D, Coulson JM, Dasgupta I, et al. A randomized controlled crossover trial evaluating differential responses to antihypertensive drugs (used as mono- or dual therapy) on the basis of ethnicity: the comparIsoN oF Optimal Hypertension RegiMens; part of the Ancestry Informative Markers in HYpertension program-AIM-HY INFORM trial. Am Heart J. 2018;204:102-8.

10. Hoy SM. Patisiran: first global approval. Drugs. 2018;78:1625-31.

11. Ray KK, Landmesser U, Leiter LA, Kallend D, Dufour R, Karakas M, et al. Inclisiran in patients at high cardiovascular risk with elevated LDL cholesterol. N Engl J Med. 2017;376:1430-40.

12. Uijl E, Mirabito Colafella Katrina M, Sun Y, Ren L, van Veghel $\mathrm{R}$, Garrelds Ingrid $\mathrm{M}$, et al. Strong and sustained antihypertensive effect of small interfering RNA targeting liver angiotensinogen. Hypertension. 2019;73:1249-57.

13. Vazquez J, Correa de Adjounian MF, Sumners C, Gonzalez A, Diez-Freire C, Raizada Mohan K, et al. Selective silencing of angiotensin receptor subtype 1a (AT1aR) by RNA interference. Hypertension. 2005;45:115-9.

14. Storch U, Forst AL, Philipp M, Gudermann T, Mederos y Schnitzler M. Transient receptor potential channel 1 (TRPC1) reduces calcium permeability in heteromeric channel complexes. $\mathbf{J}$ Biol Chem. 2012;287:3530-40.

15. Hermida RC, Crespo JJ, Dominguez-Sardina M, Otero A, Moya A, Rios MT, et al. Bedtime hypertension treatment improves cardiovascular risk reduction: the Hygia Chronotherapy Trial. Eur Heart J. 2019:ehz754.

16. Rorie DA, Flynn RWV, Mackenzie IS, MacDonald TM, Rogers A. The Treatment In Morning versus Evening (TIME) study: analysis of recruitment, follow-up and retention rates postrecruitment. Trials. 2017;18:557.

17. Souto EB, Campos JC, Filho SC, Teixeira MC, Martins-Gomes C, Zielinska A, et al. 3D printing in the design of pharmaceutical dosage forms. Pharm Dev Technol. 2019;24:1044-53.

18. Neter Judith E, Stam Bianca E, Kok Frans J, Grobbee Diederick E, Geleijnse Johanna M. Influence of weight reduction on blood pressure. Hypertension. 2003;42:878-84.

19. Kent L, Morton D, Rankin P, Ward E, Grant R, Gobble J, et al. The effect of a low-fat, plant-based lifestyle intervention (CHIP) on serum HDL levels and the implications for metabolic syndrome status-a cohort study. Nutr Metab. 2013;10:58.

20. Alexander S, Ostfeld RJ, Allen K, Williams KA. A plant-based diet and hypertension. J Geriatr Cardiol 2017;14:327-30.

21. Portune KJ, Benítez-Páez A, Del Pulgar EMG, Cerrudo V, Sanz Y. Gut microbiota, diet, and obesity-related disorders - the good, the bad, and the future challenges. Mol Nutr Food Res. 2017;61:1600252.

22. Leshem A, Horesh N, Elinav E. Fecal microbial transplantation and its potential application in cardiometabolic syndrome. Front Immunol. 2019;10:1341.

23. Ponte Márquez PH, Feliu-Soler A, Solé-Villa MJ, Matas-Pericas L, Filella-Agullo D, Ruiz-Herrerias M, et al. Benefits of mindfulness meditation in reducing blood pressure and stress in patients with arterial hypertension. J Hum Hypertens. 2019;33:237-47.

24. Cramer H, Sellin C, Schumann D, Dobos G. Yoga in arterial hypertension. Dtsch Arztebl Int. 2018;115:833-9.

25. Elmer PJ, Obarzanek E, Vollmer WM, Simons-Morton D, Stevens VJ, Young DR, et al. PREMIER Collaborative Research Group. Effects of comprehensive lifestyle modification on diet, weight, physical fitness, and blood pressure control: 18-month results of a randomized trial. Ann Intern Med. 2006;144:485-95.

26. Bengtsson U, Kjellgren K, Hallberg I, Lindwall M, Taft C. Improved blood pressure control using an interactive mobile phone support system. J Clin Hypertens. 2016;18:101-8.

27. Nolan Robert P, Feldman R, Dawes M, Kaczorowski J, Lynn H, Barr Susan I, et al. Randomized controlled trial of e-counseling for hypertension. Circulation. 2018;11:e004420.

28. Burnier M, Egan, Brent M. Adherence in hypertension. Circ Res. 2019;124:1124-40.

29. Thompson D, Mackay T, Matthews M, Edwards J, Peters NS, Connolly SB. Direct adherence measurement using an ingestible sensor compared with self-reporting in high-risk cardiovascular disease patients who knew they were being measured: a prospective intervention. JMIR Mhealth Uhealth. 2017;5:e76.

30. Ogink PAM, de Jong JM, Koeneman M, Weenk M, Engelen LJ, van Goor $\mathrm{H}$, et al. Feasibility of a new cuffless device for ambulatory blood pressure measurement in patients with hypertension: mixed methods study. J Med Internet Res. 2019;21:e11164.

31. Omboni S, Caserini M. Effectiveness of pharmacist's intervention in the management of cardiovascular diseases. Open Heart. 2018;5:e000687.

32. Change4life. https://www.nhs.uk/change4life (2020).

33. Beaney T, Burrell LM, Castillo RR, Charchar FJ, Cro S, Damasceno A, et al. May Measurement Month 2018: a pragmatic global screening campaign to raise awareness of blood pressure by the International Society of Hypertension. Eur Heart J. 2019;40:2006-17.

34. Tompson A, Fleming S, Lee M-M, Monahan M, Jowett S, McCartney D, et al. Mixed-methods feasibility study of blood pressure self-screening for hypertension detection. BMJ Open. 2019;9: e027986.

35. WHO. https://www.who.int/cardiovascular_diseases/global-hea rts/en/ (2016).

36. Dzau VJ, Balatbat CA. Future of hypertension. Hypertension. 2019;74:450-7. 